\title{
Composição florística de uma floresta de várzea sob influência da macrodrenagem em Belém, Pará
}

Estudos demonstraram que a tendência da ocupação urbana nas grandes cidades é consumir quantidades cada vez maiores de áreas verdes, o que vem provocando sérios danos ao ambiente natural e a saúde das populações. Para tanto, com o objetivo de avaliar os impactos negativos na composição florística, foi realizado o inventário de espécies florísticas da região. A área do estudo possui 44,06 ha e se insere no Parque Ecológico do Município de Belém distando cerca de 2 km da área aeroportuária. Na elaboração do mapa de vegetação, fez-se análise em ortofotos do aerolevantamento da cidade de Belém e utilizou-se de bases cartográficas do IBGE e levantamento topográfico. Para efeito de avaliação dos parâmetros, comparou-se o levantamento em três áreas distintas internas ao Parque. Nas áreas internas ao Parque, observou-se uma forte identidade florística entre os fragmentos traduzida nos valores de dominância, abundância e frequência das espécies. $\mathrm{Na}$ área A1 (Áreas contíguas), os valores de dominância e abundância foram 29,51m2/ha e 1.783 ind./ha, A2 (Áreas adjacentes), 30,01m2/ha e 1.723 ind./ha e A3 (Área de entorno), 31,18m2/ha e 841 ind./ha, respectivamente. Os principais descritores avaliados pelas análises fitossociológicas apontam para um ambiente rico e diversos quando comparado a outras comunidades florísticas, indicando tratar-se de uma comunidade madura com os diversos estádios de florestas na Amazônia. $\mathrm{O}$ alto grau de homogeneidade entre as parcelas indica tratar-se de um mesmo ambiente florístico, sugerindo que as perdas qualitativas em decorrência ao desmatamento na área do canal, não deva provocar alterações na diversidade florística do Parque.

Palavras-chave: Macrodrenagem; Área Urbana; Florística; Fitossociologia.

\section{Floristic composition of a lowland forest under the influence of macrodrainage in Belém, Pará}

\begin{abstract}
Studies have shown that the tendency of urban occupation in large cities is to consume increasing amounts of green areas, which has been causing serious damage to the natural environment and the health of populations. In order to evaluate the negative impacts on the floristic composition, an inventory of the region's floristic species was carried out. The study area has $44.06 \mathrm{ha}$ and is inserted in the Ecological Park of the Municipality of Belém. In the elaboration of the vegetation map, orthophotos were analyzed in the aero-survey of the city of Belém and cartographic bases of the IBGE and the topographic survey were used, dividing the area in three distinct regions. In the internal areas of the Park, a strong floristic identity was observed between the fragments, translated in the values of dominance, abundance and frequency of the species. In the Area A1, the values of dominance and abundance were $29.51 \mathrm{~m} 2 / \mathrm{ha}$ and 1,783 ind./ha, $\mathrm{A2}, 30.01$ $\mathrm{m} 2 / \mathrm{ha}$ and 1,723 ind./ha and A3 ), $31.18 \mathrm{~m} 2 / \mathrm{ha}$ and 841 ind./ha, respectively. The main parameters evaluated by the phytosociological analyzes point to a rich and diverse environment when compared to other floristic communities, indicating that this is a mature community with the different stages of forests in the Amazon. The high degree of homogeneity between the plots indicates that it is the same floristic environment, suggesting that the qualitative losses due to deforestation in the area of the macrodrainage should not cause changes in the floristic diversity of the Park.
\end{abstract}

Keywords: Macrodrainage; Floodplain; Floristics; Phytosociology.

Topic: Ciências Florestais

Reviewed anonymously in the process of blind peer
Received: $12 / 04 / 2019$

Approved: 27/05/2019
Pedro Guerreiro Martorano (ic)

Universidade Federal Rural da Amazônia, Brasil http://lattes.cnpq.br/0378495686512004 http://orcid.org/0000-0002-7882-7556 p.martorano60@ufra.edu.br

Jessivaldo Rodrigues Galvão (iD

Universidade Federal Rural da Amazônia, Brasil http://lattes.cnpq.br/0013591065769741 http://orcid.org/0000-0003-4242-6555

jessigalvao50@gmail.com

Rosemiro dos Santos Galate (it)

Universidade Federal Rural da Amazônia, Brasil http://lattes.cnpq.br/5727110693165421 http://orcid.org/0000-0002-3448-9487 rosemiro.galate@ufra.edu.br
Leonardo de Almeida Oliveira (iD)

Universidade Federal Rural da Amazônia, Brasil http://lattes.cnpq.br/8171296454284490 http://orcid.org/0000-0002-5195-6622 lenardoaaloliveira@gmail.com

\section{Joel Correa de Souza (10)}

Universidade Federal Rural da Amazônia, Brasil http://lattes.cnpq.br/2582560760025598 http://orcid.org/0000-0003-3001-1635 joel.correia@ufra.edu.br

Antonio Diego Lobo Paraense (iD

Universidade Federal Rural da Amazônia, Brasil http://lattes.cnpq.br/4736920669735562 http://orcid.org/0000-0001-5636-0353 antonioloboparaense@gmail.com
Bruno Kleidson da Silva Maia (i)

Universidade Federal Rural da Amazônia, Brasil http://lattes.cnpq.br/7499950655282322 http://orcid.org/0000-0002-8494-6834 brunokleidson@gmail.com de uma floresta de várzea sob influência da macrodrenagem em Belém, Pará. Revista Ibero Americana de Ciências Ambientais, v.10, n.3, p.8-19, 2019. DOI: http://doi.org/10.6008/CBPC21796858.2019.003.0002 


\section{INTRODUÇÃO}

A perda da cobertura vegetal natural na Região Metropolitana de Belém vem acontecendo em ritmo acelerado em especial nas regiões periféricas, devido ao fenômeno das chamadas invasões que se configura por loteamentos e ocupações irregulares, além da implantação de conjuntos habitacionais e obras de infraestrutura que se configuram em novos aterramentos para confecção de vias de acesso e obras de saneamento, entre outras.

O Projeto de Macrodrenagem da Bacia do Una levado a efeito pelo Governo do Estado do Pará e o Município de Belém, através da Companhia de Saneamento do Estado do Pará (COSANPA) se torna imprescindível para o saneamento de grande parte da cidade Belém. Se por um lado as obras de alargamento e dragagem propiciarão melhorias na qualidade de vida das populações beneficiadas, por outro lado deverão impactar de forma negativa a cobertura vegetal natural que ali se faz, contribuindo assim de forma decisiva para uma maior fragmentação de vegetação urbana da cidade, ao término das obras.

O desmatamento para atender as necessidades das obras não apenas atingiu as áreas legalmente preservadas assim com aquelas ditas 'Institucionais' pertencentes ao Ministério da Marinha e a Companhia das Docas do Pará (CDP). Parte deste desmatamento foi destinado à construção de conjuntos residenciais e implantação das pistas marginais ao canal.

O adensamento populacional foi, gradativamente, destruindo as áreas verdes da Região Metropolitana de Belém, com a expansão da malha viária, infraestrutura física e começo da macrodrenagem de áreas de várzea. Por isso, surgiram os parques urbanos sob uma visão de fornecer as cidades espaços adequados atendendo a uma nova demanda social, ou seja, criando um espaço para o lazer e o tempo do ócio, fugindo da realidade urbana agitada (CARDOSO et al., 2015).

A cidade de Belém, nas últimas décadas vem apresentando significativo crescimento urbano, até certo ponto carente de planejamento adequado. Um aspecto característico é o aumento da densidade de estruturas prediais verticais e a expansão horizontal indiscriminada com perda de vegetação. Nesta cidade com o processo de urbanização há modificações no solo, este originalmente vegetado, dar espaço para uma superfície compactada e impermeabilizada, por estar na presença de construção civil, pavimentação, entre outros processos artificiais (BRAGA, 2017).

Historicamente o Parque Ecológico do Município de Belém (PEMB), local da realização dos estudos, teve sua área preservada graças às ações conservacionistas de moradores das áreas residenciais próximas, preservando-as, e promovendo ações que posteriormente resultaram no seu tombamento como Parque. A falta de fiscalização e os fatores culturais relacionados ao uso dos recursos naturais como coleta de frutos, a área do Parque vem passando diversos processos antrópicos por parte da população do entorno tais como a retirada de madeira, caça e pesca ilegal.

No contexto, é imprescindível o estudo florístico pois, a partir das informações quali-quantitativas adquiridas do estudo, será possível conhecer a função de diversas espécies de plantas na comunidade, além de identificar seus habitats preferenciais. O objetivo deste estudo visa a caracterização da composição 
florística arbórea da vegetação, suas perdas, avaliação do grau de alteração na florística e da regeneração natural em áreas não alteradas, bem como nas áreas do entorno traduzidos pelos índices de diversidade e similaridade florística.

\section{MATERIAIS E MÉTODOS}

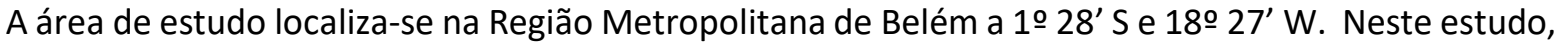
considerou-se uma área totalizando 44,06 ha (Figura 1).

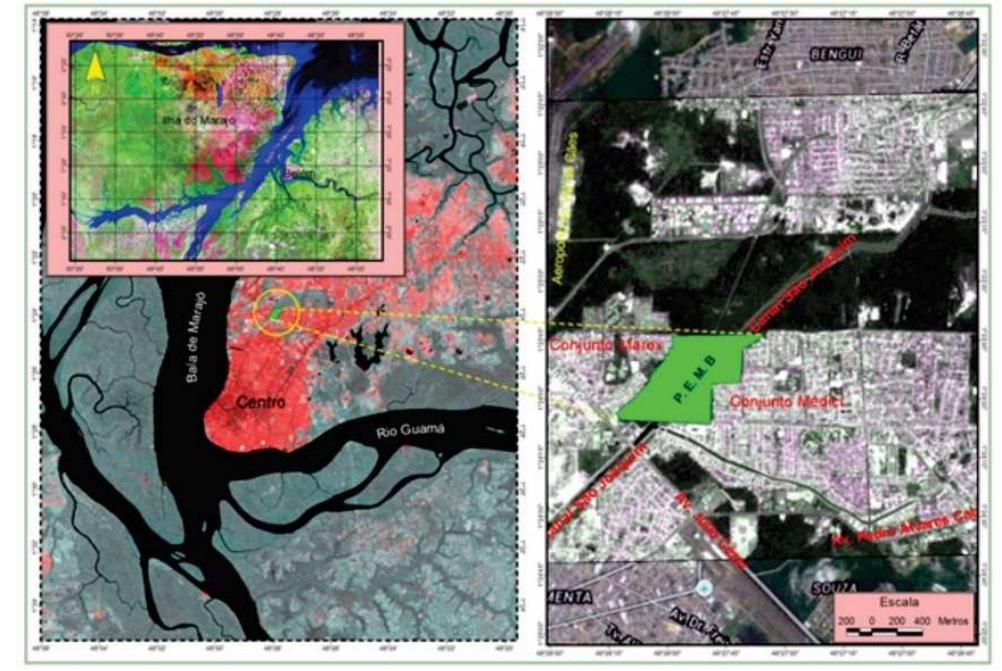

Figura 1: Espaço geográfico em que se localiza o Parque Ecológico do Município de Belém (PEMB).

Legalmente, a área do Parque Ecológico do Município de Belém (PEMB) apresenta somente 33,30ha (Lei Municipal ํ 7.553 de 19 de novembro de 1991). O restante da área 10,76 ha, mesmo não estando legalmente tombado por questões de ordens formais, também foi incluída no estudo. A caracterização climática é do tipo Af, segundo Koppen, com clima tropical chuvoso, precipitação média mensal maior que 60mm e com baixa amplitude térmica anual (BARBOSA et al., 2015).

O solo apresenta na sua parte superficial textura argilosa ou média, com características próprias de solos de várzea. A sequência sedimentar é essencialmente argilosa, caracterizados como gleissolos álicos, como na maior parte da área, ou como gleissolos eutróficos, caracterizados nas margens de alguns igarapés (EMBRAPA, 2018).

Utilizaram-se parcelas amostrais de $10 \mathrm{~m} \times 15 \mathrm{~m}\left(150 \mathrm{~m}^{2}\right)$ que foram assim distribuídas: um total de 49 parcelas para as áreas contiguas (A1- próximo ao canal), 65 parcelas para áreas adjacentes (A2) e 13 para áreas do entorno (A3), que totalizaram 7.350, 9.750 e $1.950 \mathrm{~m}^{2}$ respectivamente, representando 4,31\% de incidência amostral. Ressalta-se que se avaliou ainda parcelas fora da área do Parque (próximas), para estabelecer parâmetros comparativos a nível de comunidades florísticas.

As coletas foram realizadas de maio a setembro, período em que foram processadas as exsicatas do material fértil das espécies para identificação das espécies que precisavam de melhor identificação. As exsicatas foram referenciadas junto ao Herbário do Museu Emílio Goeldi. O sistema adotado para a classificação do material foi o de Cronquist (1981). 
Para a determinação da área mínima representativa e do tamanho das parcelas, considerando que as áreas apresentam vegetação em estádio sucessional relativamente uniforme, utilizou-se a curva espécieárea (MATTEUCCl et al., 1982). O método adotado indica que é possível obter-se um tamanho ideal para uma boa amostragem na medida em que se aumenta o tamanho da unidade da amostra. Nas primeiras amostras ocorre um aumento brusco do número de espécies até que chegando a determinado ponto, o acréscimo de novas espécies começa a ser muito baixo ou nulo.

Os índices de diversidade mais utilizados incluem o índice de Simpson e o índice de Shannon-Wiener, dos quais o de Simpson é mais sensível a mudanças nas espécies mais abundantes e o de Shannon-Wiener é mais sensível a mudanças nas espécies raras da comunidade, sendo mais utilizado em programas de manejo e conservação. Neste trabalho, utilizou-se o índice de Shannon- Wiener (H'), utilizado por Poole (1974), e Equabilidade (PIELOU, 1975). Segundo Brower et al. (1977), o índice de dominância de Simpson (C) mostra a probabilidade de que dois indivíduos em uma comunidade de $\mathrm{N}$, sendo tomados aleatoriamente, ambos, sejam da mesma espécie sendo dado por:

$$
\begin{gathered}
\mathrm{S} \\
C=\sum\left[n_{i}\left(n_{i}-1\right)\right] /[N(N-1)] \\
i-I \\
\mathrm{~S} \\
H^{\prime}=-\sum P_{i} x \log \left(p_{i}\right), \text { onde } P_{i=} n_{i} / N \\
i-I \\
J=H^{\prime} / H \max \\
I=H^{\prime} / H \max
\end{gathered}
$$

Onde: D = Índice de diversidade de Simpson; $\mathrm{J}=$ Índice de Equabilidade; $\mathrm{H}^{\prime}$ = Índice de Shannon; $\mathrm{n}_{\mathrm{i}}=$ Número de indivíduos da i-ésima espécie; $\mathrm{N}=$ Número total de indivíduos amostrados; $\log =$ Logaritmo de base 10; $\mathrm{H}^{\prime} \max =\log (\mathrm{S})$; $S=$ Número total de espécies; $p_{i}=$ Porção da abundância da i-ésima espécie em relação ao total; e $D=1-C$, onde $C$ é a medida de dominância de Simpson.

As espécies raras possuem um baixo número de indivíduos em uma comunidade. Nas amostras que envolvem o levantamento de espécies, normalmente encontra-se apenas um exemplar da espécie (BEGON, 2007). Oliveira et al. (2004) define como sendo aquelas espécies que são representadas por apenas um indivíduo na amostra, sendo este último conceito adotado neste estudo dado pela seguinte expressão:

$$
E R \%=n_{i} / N \times 100
$$

Para a análise de similaridade florística nas áreas estudadas, foram calculados os índices qualitativos de Jaccard, Sorenson e Kulczynsk dados pelas expressões abaixo, as mesmas adotadas por Oliveira (1997). 


$$
\begin{gathered}
S_{j a c}=c /(a+b+c) \\
S_{s o r}=2 c /(a+b+2 c) \\
S_{\text {kur }}=0,5[(c /(a+c)+c /(b+c))]
\end{gathered}
$$

Onde:

$S_{j a c}=$ Índice de Similaridade de Jaccard;

$S_{\text {sor }}=S_{\text {sor }}=$ indice de Similaridade de Sorenson;

$S_{\text {kur }}=S_{k u l}=$ Índice de Similaridade de Kulczynski;

$a=$ Número de táxons exclusivos da área $a$;

$b=$ Número de táxons exclusivos da área $b$; $\mathrm{e}$

$c=$ Número de táxons comuns ás áreas $a$ e $b$

O índice de Morisita-Horn $(\mathrm{CH})$ é utilizado para medir a similaridade entre duas comunidades. Pode ser interpretado como a probabilidade de que um indivíduo ao ser retirado da amostra j e outro da amostra k pertençam à mesma espécie, varia de zero a um, podendo ser empregado em dados de abundâncias, independentemente do tamanho amostral. Utilizou-se o índice de similaridade quantitativo de Morisita, simplificado por Horn (1966) dado pela expressão abaixo;

$$
S_{\text {mor }}=\left(2 \sum X i j . X i k\right) /\left[\left(\sum X i j 2 / N j 2\right)+\left(\sum X i k 2 / N k 2\right)\right] N j . N k
$$

Em que:

$S_{m o r}=$ Índice simplificado de Morisita proposto por Horn (1966); Xij e Xjk = abundâncias da i-ésima espécie das amostras j e k; Nj e $N k$ = número total de indivíduos nas amostras j e k.

A avaliação dos aspectos qualitativos do povoamento teve como principal objetivo a complementação das inferências para as análises da estrutura interna do povoamento florístico, relacionada a possíveis defeitos depreciativos quanto à qualidade do fuste, sanidade da árvore e ocorrência de impedidores ao seu bom desenvolvimento natural (Figura 2).
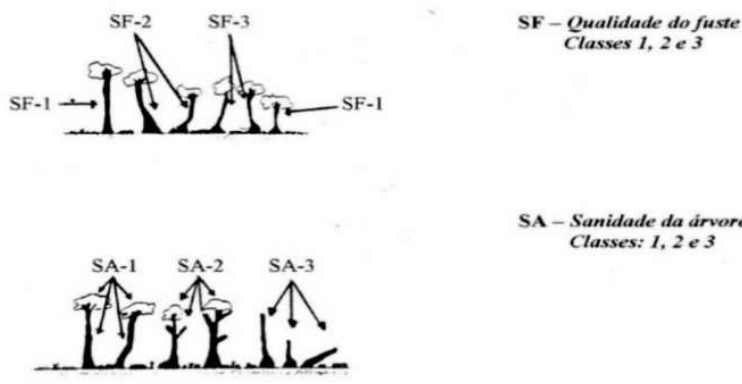

Classes: $1,2 e^{3}$

OI - Ocorrência de impedidores
Classes: 1 e 2 .

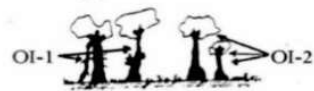

Figura 2: Componentes da avaliação por classes de sanidade da árvore, qualidade do fuste e ocorrência de impedidores de crescimento da vegetação na área do PEMB

Avaliou-se os aspectos referentes a sanidade da árvore como um todo considerando a ocorrência de deformidades apresentadas no fuste e copa que, de alguma, forma possam contribuir para uma depreciação do valor comercial. Assim, as árvores foram classificadas da seguinte forma: classe de sanidade 1 -indivíduos com boa performance de copa e fuste sem apresentar danos visíveis em qualquer das partes; classe de sanidade 2 - indivíduos apresentando perda parcial de copa por queda de galhadas e apresentando 
perfurações inicial de insetos; classe de sanidade 3 - indivíduos apresentando perda superior a metade da área de copa ou perda total de galhadas apresentando ou não ataque generalizado de insetos.

Quanto à qualidade do fuste considerou-se as seguintes classes: classe 1, fuste perfeito - Indivíduos apresentando pouca inclinação ou sulcamentos e possibilidade de aproveitamento industrial integral, classe 2, fuste tortuoso - indivíduos apresentando inclinação acentuada, tortuosidade e nodosidade com possibilidade de aproveitamento industrial parcial; classe3, fuste muito tortuoso - indivíduos cujas deformidades o inviabilizam para o aproveitamento industrial porém, podendo ter utilização para fins energéticos e/ou domésticos (carvão, lenha).

Quanto a ocorrência de impedidores de crescimento: classe 1, epífitas e cipós ausentes - indivíduos apresentando ausência de impedidores no fuste e copa por cipós lenhosos ou herbáceos; classe 2, epifitas e cipós presentes - Indivíduos apresentando ocorrência de epífitas e cipós ocupando parcialmente ou totalmente fuste e copa incluídos os chamados 'mata paus'.

\section{RESULTADOS E DISCUSSÃO}

Foram amostrados 3.160 indivíduos numa área total de 1,90ha de uma floresta de várzea sendo: 1.313 indivíduos pertencentes a área Al (Contíguas ao canal), 1.683 pertencentes a área A2 (Área adjacentes ao canal) e 164 pertencentes a área A3 (Área do entorno do canal, mata da Marinha). A população de indivíduos está distribuída em 21 famílias, 42 gêneros e 48 espécies, para o nível de abordagem com CAP $\geq$ $15 \mathrm{~cm}$.

Das espécies inventariadas, 21 são exclusivas do ambiente, sendo 3 espécies na área A1, dez espécies na área A2 e oito espécies na área A3 conforme verifica-se na tabela 3. A ocorrência de espécies raras é caracterizada por espécies que se fizeram representar por apenas um indivíduo na amostra. Assim para a área A1(Canal), A2 (Adjacentes) e A3 (Entorno) os índices obtidos foram 27,7\%, 28,5\% e 37,7\% respectivamente. Esse resultado demonstra haver uma tendência de aumento na riqueza florística à medida que ocorre a elevação das cotas do terreno no sentido várzea/terra firme.

Os valores de dominância $\left(\mathrm{m}^{2} \mathrm{ha}^{-1}\right)$ e abundância ( $\mathrm{N} /$ ind.há $\left.^{-1}\right)$ para as áreas consideradas, estão assim distribuídos: área Al - (19,51 m²há-1 e 1.786 ind.há $\left.^{-1}\right)$; área A2 (20,01 m² ha-1 e 1.735 ind.ha-1); área A3 (21,18 há ${ }^{-1}$ e 863 ind. $\mathrm{Ha}^{-1}$ ) respectivamente. $\mathrm{Na}$ área $\mathrm{Al}$, considerando-se os dois níveis de abordagem, oito das dezessete famílias que ocorrem são responsáveis por $66,6 \%$ das espécies catalogadas na área do Parque. As famílias são Clusiaceae, Arecaceae, Fabaceae, Myristicaceae, Lecythidaceae, Mimosaceae, Caesalpiniaceae e Ebenaceae. Somente as duas primeiras famílias, que representam $11,7 \%$ do total das famílias, contribuíram com 22,2\% das espécies da área (Tabela 1 ).

$\mathrm{Na}$ área $\mathrm{A} 2$, oito das dezenove famílias contribuíram com $50 \%$ das espécies catalogadas. As famílias são Clusiaceae, Arecaceae, Fabaceae, Mirysticaceae, Mimosaceae, Lecytidaceae, Anonaceae e Ebenaceae. Se considerarmos as duas primeiras famílias as quais representam somente $10,52 \%$ do total, estas contribuem com $25 \%$ das espécies (Tabela 1 ). 
Tabela 1: Famílias e espécies com $C A P \geq 15 \mathrm{~cm}$ para os níveis de abordagem I e Il da população nas áreas A1 - contíguas, $A 2$ - adjacentes e $A 3$ - do entorno que ocorrem na área do PEMB

\begin{tabular}{|c|c|c|c|c|c|c|c|c|}
\hline \multirow{2}{*}{ Família } & \multirow{2}{*}{ Espécie } & \multirow{2}{*}{ Nome vulgar } & \multicolumn{3}{|c|}{ NI A BRD I } & \multicolumn{3}{|c|}{ NI A BRD II } \\
\hline & & & A1 & A2 & A3 & A1 & A2 & A3 \\
\hline Anacardiaceae & Tapirira guinensis & Tatapiririca & & & & $x$ & $x$ & $x$ \\
\hline Anonnaceae & Xybpia nittda Dun. & Envira cana & $x$ & $\mathrm{x}$ & & $\mathrm{x}$ & $\mathrm{x}$ & $x$ \\
\hline Apocinaceae & Himatanthus sp & Bichi-bichi & $x$ & $x$ & & $x$ & $x$ & $x$ \\
\hline \multirow[t]{7}{*}{ Arecaceae } & Astrocarium vulgare Mart. & Tucumã-i & & & & & & $x$ \\
\hline & Euterpe oleracea & Açaí & $x$ & $x$ & $x$ & $x$ & $x$ & $x$ \\
\hline & Mauritia armata Mart. & Buriti & & & & & $\mathrm{x}$ & \\
\hline & Maximiliana regia & Inajá & & & & & & $x$ \\
\hline & Maurutia 14araná Wall. & Caranã & $x$ & $x$ & $x$ & $x$ & $x$ & $x$ \\
\hline & Oenorcarpus distilus Mart. & Bacaba & & & & & & $\mathrm{x}$ \\
\hline & Socratea exotiza Wenol & Paxiúba & & & $x$ & & $x$ & $x$ \\
\hline \multirow[t]{2}{*}{ Bombacaceae } & Bombax paraenses & Mamorana & & & & & & $x$ \\
\hline & Bombax globosum & Mugumba & & $x$ & & & $x$ & \\
\hline \multirow[t]{2}{*}{ Burseraceae } & Protium sagothianum March. & Breu branco & & & & $x$ & $x$ & \\
\hline & Protium sp & Breu preto & & & & & $\mathrm{x}$ & \\
\hline \multirow[t]{2}{*}{ Caesalpiniaceae } & Dimorphanda macrostachia & Faveiro de pombo & & & & $\mathrm{x}$ & $x$ & $x$ \\
\hline & Macrobbium acaciaefolium Bht. & Arapari & & & & $x$ & & \\
\hline \multirow[t]{2}{*}{ Cariocareceae } & Cariocar macrocarpum (Aubp Pers) & Piquiarana & & & & & $x$ & $\mathrm{x}$ \\
\hline & Cariocar vilosum Aubl & Piquiá & & & & & $\mathrm{x}$ & \\
\hline \multirow[t]{2}{*}{ Cecropiaceae } & Cecropia fisifolia Snethi & Embauba branca & & & & $\mathrm{x}$ & $x$ & $x$ \\
\hline & Cecropia sp & Embauba & & & & & $x$ & \\
\hline Crysobalanaceae & Licania hteromorpha Bth & Macucu & & & & & $x$ & \\
\hline Ebanaceae & Diospirus melinonni (Hierne) Ac. Smith & Caqui & $\mathrm{x}$ & $\mathrm{x}$ & & $\mathrm{x}$ & $\mathrm{x}$ & \\
\hline Erythroxylaceae & Erythroxillum kapplerianum & Passarinheira & & & & & $x$ & \\
\hline Euphorbiaceae & Hevea brasiliensis (H.B.K) Muell. Arg. & Seringeira & & & & $x$ & $x$ & $x$ \\
\hline \multirow[t]{7}{*}{ Fabaceae } & Andira retusa (L.A.M.) K.B.K. & Andira-uchi & & & & & $x$ & \\
\hline & Cassia letrandra & Fava marimari & & & & & & $x$ \\
\hline & Dipterix odorata & Cumaru & & & & $\mathrm{x}$ & & \\
\hline & Macrobbium bifolium (Aubi.) Pers & Iperana & $x$ & & $x$ & $x$ & $x$ & $x$ \\
\hline & Ormosia coutinhoi (Duck.) & Buiuçu & & $x$ & $x$ & & $x$ & $x$ \\
\hline & Pterocarpus amazonicus Huber. & Mututi & & & & $\mathrm{x}$ & $\mathrm{x}$ & \\
\hline & Taralea oppositifolia Aubl. & Cumarurana & $x$ & $x$ & $x$ & $x$ & $x$ & $x$ \\
\hline \multirow[t]{6}{*}{ Clusiaceae } & Clusia grandifflora & Apui & & & & $\mathrm{x}$ & $x$ & \\
\hline & Calophyllum brasiliensis Comb. & Jacabeúba & $x$ & $\mathrm{x}$ & $\mathrm{x}$ & $\mathrm{x}$ & $\mathrm{x}$ & $\mathrm{x}$ \\
\hline & Caraipa grandiflora Mart. & Tamaquaré & $\mathrm{x}$ & $\mathrm{x}$ & & $\mathrm{x}$ & $x$ & $x$ \\
\hline & Chaumochiton kaplei (S. ex engel) Duck. & Lacrão & & & & & $x$ & $x$ \\
\hline & Symphonia gbbulifera L.F. & Anani & $x$ & $x$ & $x$ & $\mathrm{x}$ & $x$ & $x$ \\
\hline & Tovomita brasiliensis (Mart.) Warp. & Manguirana & $x$ & & & $\mathrm{x}$ & & \\
\hline \multirow[t]{2}{*}{ Lecythidaceae } & Alantoma lineata (Berg) Wurb. & Xuru & $x$ & $x$ & & $x$ & $x$ & $x$ \\
\hline & Couratari sp & Tauari & & & & & & $x$ \\
\hline \multirow[t]{2}{*}{ Meliaceae } & Carapa guinensis Aubl. & Andiroba & & & & & $x$ & \\
\hline & Trichilia leicontei Duck. & Caxuá & $x$ & & & $\mathrm{x}$ & $x$ & $x$ \\
\hline \multirow[t]{3}{*}{ Mimosaceae } & Enterobium maximum Duck. & Fava bolacha & $x$ & $x$ & $x$ & $\mathrm{x}$ & $x$ & $x$ \\
\hline & Inga sp Willd. & Inga pretinho & & & & & $x$ & \\
\hline & Pithecelobium jupumba (Wiki) Urb. & Saboeiro & & & & $\mathrm{x}$ & $x$ & $x$ \\
\hline Mirysticaceae & Virola surinamensis (pol.) Warb & Virola & $x$ & $x$ & $x$ & $x$ & $x$ & $x$ \\
\hline \multirow[t]{2}{*}{ Moraceae } & Brosimum parinoides & Amapá doce & & & & & & $\mathrm{x}$ \\
\hline & Ficus antihelminthia Willd. Var. incipida & Caxinguba & & & & $x$ & & \\
\hline Vochysiaceae & Vochysia guinensis & Quarubatinga & & & & & & $x$ \\
\hline
\end{tabular}

$\mathrm{Na}$ área $\mathrm{A} 3$, oito das dezesseis famílias contribuíram com $72,4 \%$ das espécies. As famílias são Arecaceae, Fabaceae, Clusiaceae, Miristycaceae, Caesalpiniaceae, Lecythidaceae, Moraceae e Mimosaceae. Considerando-se as duas primeiras famílias as quais representam 11,76\%, estas contribuíram com 34,48\% das espécies (Tabela 1).

Lau et al. (2013), estudando a composição florística de uma floresta de várzea da Reserva de Proteção Ambiental da llha de Combu, no município de Belém, encontrou 5.731 indivíduos distribuídos em 21 famílias, 
51 gêneros, 61 espécies. As famílias com maior número de espécies foram Fabaceae (14 espécies) e Arecaceae (7 espécies) e com maior número de indivíduos Arecaceae (4.364), Malvaceae (374) e Fabaceae (349) enquanto as outras 18 famílias somaram 644 indivíduos, sendo a espécie mais representativa a Euterpe oleracea.

Em estudos na floresta de várzea do município de Breves, Pará, as espécies Symphonia globulifera, Carapa guianensis, Virola surinamensis e Pentaclethra macroloba apresentaram número de indivíduos, frequência e dominância relativa com valores acima daqueles registrados para E.oleracea (JARDIM et al., 2004).

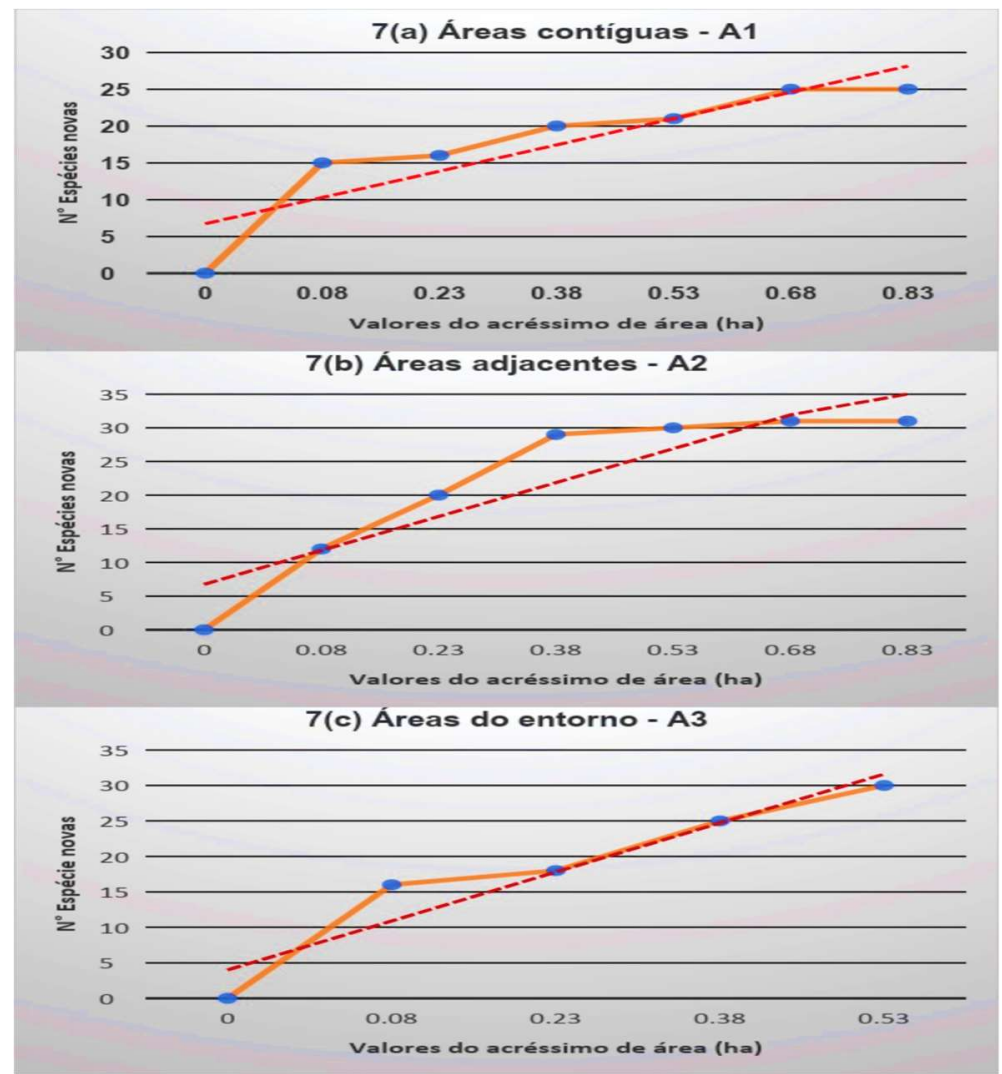

Figura 3: Curva cumulativa de espécies adicionais (espécie-área) para as áreas contíguas (A1 - Figura 3a); áreas adjacentes (A2 - Figura 3b) e área de entorno (A3 - Figura $3 c$ ) no PEMB

Ayres (1995), estudando as várzeas do Mamirauá (Baixo Japurá), comprovou haver uma correlação entre o aumento da riqueza florística e a elevação das cotas do terreno. Na restinga alta foram encontradas 135 espécies e na restinga baixa apenas 109. Também comprovou ser este gradiente um fator determinante para a composição das espécies, dominância e abundância uma vez que este determina a fertilidade do solo, drenagem e duração das inundações.

Estudos diversificados têm demonstrado a relação entre as variáveis topográficas com a diversidade e distribuição de espécies vegetais na paisagem (KIRKBY et al., 1990; BISPO et al., 2009). As colocações destes autores se juntam à influência sobre a vegetação através de efeitos do relevo sobre a hidrologia, uma vez que ele determina o traçado planimétrico do curso de rios, variações na disponibilidade de água no solo e distribuição dos tipos de solo.

Analisou-se o comportamento da curva espécie-área conforme consta na figura 4 e levando-se em consideração três comunidades estudadas Al (Canal), A2 (Adjacentes) e A3 (Entorno) como forma de explicar 
a abordagem florística e a suficiência amostral. No que se refere as áreas Al e A2 (Figuras 3a e 3b), estas apresentaram um comportamento muito próximo quanto a ascensão e inflexão da curva, o mesmo não acontecendo na área A3 (Figura 3c). Para as duas primeiras, do marco zero até 0,30 ha foi observado um crescimento inicial brusco da curva e após este, um crescimento de maneira menos acentuada até alcançar 0,60 ha no eixo dos ' $X$ ', ponto em que ocorreu a inflexão quase que horizontal da curva. Esse comportamento demonstra a suficiência amostral no que se refere ao tamanho da amostra indicando que a amostragem foi suficiente para abranger a maioria das espécies das referidas comunidades (Figura 4a e 4b) sendo capaz de explicar a riqueza florística nas áreas referidas.

$\mathrm{Na}$ área $\mathrm{A} 3$, do ponto zero até o ponto 0,30 ha, não houve a inflexão da curva indicando, portanto que o acréscimo de novas áreas não determinou a estabilização que permitisse a inflexão da curva de onde se conclui que a amostragem não foi suficiente para representar a comunidade em questão. Tal comportamento já era esperado pois pretendeu-se apenas estabelecer alguns parâmetros comparativos com as demais comunidades inferidas, uma vez se tratar de uma área com maior proximidade com a mata de terra firme, o que a caracteriza como transição (Figura 3c).

Os valores encontrados neste estudo estão em concordância com os autores citados, assim como no que se refere ao dimensionamento da área abrangida. Observou-se que as primeiras espécies levantadas, foram as mais abundantes uma vez que estas se fizeram presentes numericamente bem antes de atingir a inflexão da curva, que no estudo foi de 0,6 ha, sendo que foram também as mais abundantes, a exemplo das espécies Mauritia carana, Caraipa grandiflora e Symphonia globulifera.

Nos estudos realizados por Rabelo (1999), observa-se que nas localidades estudadas, Mazagão apresentou tendência de ocorrer a inflexão da curva após o segundo hectare, com aproximadamente 50 espécies, enquanto que na localidade do Lontra para atingir o mesmo número de espécies, foi necessário percorrer o dobro da área, ou seja, 4 ha. Já para a área do Parque isso não ocorreu, uma vez que nas áreas contíguas (A1) e adjacentes (A2) as quantidades de novas espécies ocorreram em áreas quase que equivalentes (Figuras 3a e 3b). As similaridades florísticas foram descritas de acordo com Jaccard, Sorenson, Kulszynski e Morizita-Horn (Tabela 2). Considerando os valores comparativos obtido ao nível de Espécie, Gênero e Família, as áreas que apresentaram os maiores valores de similaridade, correspondem as áreas A1 e A2.

Também é possível identificar que da área A1 para a área A3 existe uma diminuição da similaridade florística. Nas áreas internas ao Parque (Al e A2) e do entorno (A3) verificou-se uma alternância decorrente dos métodos utilizados Jaccard, Sorenson, Kulszynski e Morisita-Horn. Nas áreas internas (A1/A2), em valores médios considerando os descritores acima, à similaridade a nível de espécie ficou próximo a 75\%, o mesmo valor observado para as áreas A2/A3. Nas áreas Al/A3 o valor foi de $71 \%$. Considerando o Parque (área remanescente, A2) como um todo e Colares, esse valor foi de $36 \%$. Considerando a similaridade em termos de gênero e família e os descritores para Soreson, Kulszynski e Morisita-Horn, pode-se dizer que houve um aumento desses valores no sentido gênero/família. 
Tabela 2: Índice de Similaridade florística (Jaccard, Sorenson, Kulszynski e Morizita-Horn) para os diferentes níveis taxonômicos na área do PEMB

\begin{tabular}{|l|l|l|l|l|}
\hline \multicolumn{1}{|c|}{ Ándices } & A1-A2 & \multicolumn{2}{c|}{ A2-A3 } & Parque - Colares \\
\hline Espécies & 0,60 & 0,67 & 0,68 & 0,24 \\
\hline Jaccard & 0,75 & 0,59 & 0,62 & 0,34 \\
\hline Sorenson & 0,99 & 0,88 & 0,98 & 0,55 \\
\hline Kulszynski & 0,64 & 0,70 & 0,73 & 0,32 \\
\hline Morizita-Horns & 0,68 & 0,42 & 0,47 & 0,22 \\
\hline Gênero & 0,81 & 0,59 & 0,64 & 0,36 \\
\hline Jaccard & 0,99 & 0,89 & 0,99 & 0,57 \\
\hline Sorenson & 0,70 & 0,72 & 0,31 \\
\hline Kulszynski & 0,61 & \multicolumn{2}{l|}{} \\
\hline Morizita-Horns & 0,58 & 0,52 & 0,39 \\
\hline Famílias & 0,89 & 0,69 & 0,56 \\
\hline Jaccard & 0,94 & 0,73 & 0,83 \\
\hline Sorenson & 0,99 & 0,99 & 0,73 \\
\hline Kulszynski & 0,95 & & \\
\hline Morizita-Horns & 0,98 & & \\
\hline $\begin{array}{l}\text { Área A1 (Contigua ou Canal) } \\
\text { Área A2 (Adjacente ou Remanescente) } \\
\text { Área A3 (De Entorno ou Marinha) }\end{array}$ \\
\hline
\end{tabular}

Oliveira (1997) estudando uma floresta de terra firme próximo à Manaus, observou ter esta as mesmas características quando da avaliação de área próximas (hectares 1,2 - 1,3 e 2,3) uma vez ter utilizado os mesmos descritores. Também encontrou valores que indicaram uma grande concordância para o último descrito (Morisita-Horn), ao nível taxonômico de família.

Os resultados discutidos neste estudo estão concordantes com os de Rabelo (1999), que encontrou valores de similaridade (Sorenson) para espécie em torno de $70 \%$ ao comparar duas florestas estuarinas nas localidades de Mazagão e Lontra da Pedreira, no Amapá. Para a Diversidade ( $\mathrm{H}^{\prime}$ ) e Equabilidade (J) florísticas (Tabela 3), observa-se que houve um aumento gradual da diversidade entre as áreas, resultado que apresenta coerência com os apresentados na figura 3, em que ficou evidenciada uma maior riqueza de espécies iniciais na área $\mathrm{A} 3$, por unidade de área.

Tabela 3: Índices de Diversidade Shannon ( $\left.\mathrm{H}^{\prime}\right)$, Simpson (1-D) e Equabilidade (J) florística para as áreas A1 - contíguas, $A 2$ - adjacentes e $A 3$ - do entono na área do PEMB

\begin{tabular}{|l|l|l|l|l|l|}
\hline \multirow{2}{*}{ Áreas } & \multicolumn{1}{c|}{$\begin{array}{c}\text { Shannon } \\
\left(\mathbf{H}^{\prime}\right)\end{array}$} & \multicolumn{1}{c|}{$\mathbf{C}$} & \multicolumn{1}{c|}{$\mathbf{1 / C}$} & \multicolumn{1}{|c|}{ 1-C } & \multirow{2}{*}{ Equabilidade (J) } \\
\cline { 2 - 5 } & 2,45 & 0,118 & 8,501 & 0,882 & 0,685 \\
\hline A2 & 2,53 & 0,115 & 8,678 & 0,885 & 0,678 \\
\hline A3 & 2,59 & 0,09 & 11,076 & 0,91 & 0,818 \\
\hline A1 - Área 1 (Contíguas), A2 - Área 2 (Adjacentes), A3 - Área 3 (Entorno) \\
\hline
\end{tabular}

Verifica-se que o índice de concentração de Simpson (C) para as áreas avaliadas é considerado baixo $0,12 \%$ ( $\mathrm{Al}$ e $\mathrm{A} 2$ ) e $0,10 \%(\mathrm{~A} 3)$ de onde se deduz que as espécies mais abundantes não garantem igual proporção em valores de dominância para a comunidade abordada.

\section{CONCLUSÕES}

A incorporação dos valores obtidos através da inclusão do (IVIAr\%) dados pelos valores da posição sociológica das espécies nos estratos da floresta considerando o plano vertical inferior, médio e superior assim como os valores da regeneração natural, da ocorrência de novas plântulas no sub-bosque, capaz de 
identificar as espécies que ganharam importância na comunidade florística foram Caranã (Mauritia carana); o Ipê-da-várzea (Macrolabium bifolium); O açaí (Euterpe oleraceae); e o Cumarurana (Taralea oppositifolia) o que vem demonstrar que são essas as espécies mais aptas na adoção de manejo silvicultural uma vez que estas encontram representantes tanto em regeneração quanto nos estratos da floresta também podendo incluir nessa lista as espécies Tamaquaré (Caraipa grandiflora); a Ucuuba (Virola surinamensis); o Anani (Symphonia globulifera).

No que concerne à valores que expressam a diversidade, similaridade, riqueza e ocorrência de espécies raras identificam um ambiente cuja comunidade florística se apresenta bastante diversas apresentando elevada riqueza de poucas espécies características intrínsecas a ambientes submetidos a stress hídrico típico das terras baixas inundáveis classificas como várzeas do estuário amazônico. A alta similaridade florística identificada comparativamente com as áreas analisadas indicam tratar-se de uma mesma formação tipológica, ou seja, a 'Mata da Várzea'.

\section{REFERÊNCIAS}

AYRES, J. M.. As matas de várzea do Mamirauá: médio Rio Solimões. 2 ed. Brasília: Mamirauá, 1995.

BARBOSA, P. H. D.; COSTA, A. C. L.; CUNHA, A. C.; SILVA JUNIOR, J. A.. Variabilidade de elementos meteorológicos e de conforto térmico em diferentes ambientes na Amazônia brasileira. Revista Brasileira de Climatologia, v.17, p.98-118, 2015.

BEGON, M.; TOWNSEND, C. R.; HARPER, J. L.. Ecologia: de indivíduos a ecossistemas. Porto Alegre: Artmed, 2007.

BISPO, P. C.; VALERIANO, M. M.; KUPLICH, T. M.. Relation of local geomorphometric variables with the vegetation of the Madeira-Purus interfluve (AM/RO). Acta Amazônica, v.39, n.1, p.81-90, 2009.

BRAGA, T. G. M.. A urbanização e o comportamento da temperatura superficial: uma análise temporal da região Metropolitana de Belém. Dissertação (Mestrado) Universidade Federal do Pará, Belém, 2017.

BROWER, J. E.; ZAR, J. H.. Field and Laboratory Methods for General Ecology. Wm. C. Brown Company. 1977.

CARDOSO, S. L. C.; VASCONCELLOS SOBRINHO, M; VASCONCELLOS, A. M. A.. Gestão ambiental de parques urbanos: o caso do Parque Ecológico do Município de Belém Gunnar Vingren. Revista Brasileira de Gestão Urbana, v.7, n.1, p.74-90, 2015.

CRONQUIST, H.. Na integrated system of classification of flowering plants. New York: Columbia University Press, 1981.

EMBRAPA. Empresa Brasileira de Pesquisa Agropecuária. Centro Nacional de Pesquisa de Solos: Sistema brasileiro de classificação de solos. Brasília: Embrapa, 2018.

HORN, H. S.. Measurement of 'overlap' in comparative ecological studies. The American Naturalist, v.100, p.419424, 1966.
JARDIM, M. A. G.; AMARAL, D. D.; SANTOS, G. C.; MEDEIROS, T. D. S.; SILVA, C. A.; FRANCEZ, D. C.; NETO, S. V. C.. Análise florística e estrutural para avaliação da fragmentação nas florestas de várzea do estuário amazônico. In: JARDIM, M. A. G.; MOURÃO, L.; GROISSMAN, M.. Açaí, possibilidades e limites para o desenvolvimento sustentável no estuário amazônico. Belém: Museu Paraense Emílio Goeldi, 2004. p.101-121

KIRKBY, M.; ATKINSON, K.; LOCKWOOD, J. G.. Aspect vegetation cover and erosion on semi-arid hillslopes. In: THOMES, J.. Vegetation and Geomorphology. New York: John Wiley, 1990. p.25-39.

LAU, A. V.; JARDIM, M. A. G.. Florística e estrutura da comunidade arbórea em uma floresta de várzea na Área de Proteção Ambiental, Ilha do Combu, Belém, Pará. Biota Amazônica, Macapá, v.3, n.2, p.88-93, 2013.

MATTEUCCI, S. D.; COLMA, A.. Metodologia para el estudo de la vegetacion. Washington: General Secretariat of the Organization of Américan States, 1982.

OLIVEIRA, A. A.. Diversidade, estrutura e dinâmica do componente arbóreo de uma floresta de terra firme de Manaus, Amazonas. Tese (Doutorado em Botânica) Instituto de Biociências da Universidade de São Paulo, São Paulo, 1997.

OLIVEIRA, A. N.; AMARAL, I. L.. Florística e fitossociologia de uma floresta de vertente na Amazônia Central, Amazonas, Brasil. Acta Amazônica, v.34, n.1, p.21-34, 2004.

PIELOU, E. C.. Ecological Diversity. New York: John Wiley \& Sons, 1975

POOLE, R. W.. An introduction to qualitative ecology. New York: McGraw-Hill, 1974.

RABELO, F. G.. Composição Florística, Estrutura e Regeneração de Ecossistemas Florestais na Região 
Estuarina do Rio Amazonas-Amapá-Brasil. Dissertação

(Mestrado) - Faculdade de Ciências Agrárias do Pará, Belém,

1999.

A CBPC - Companhia Brasileira de Produção Científica (CNPJ: 11.221.422/0001-03) detém os direitos materiais desta publicação. Os direitos referem-se à publicação do trabalho em qualquer parte do mundo, incluindo os direitos às renovações, expansões e disseminações da contribuição, bem como outros direitos subsidiários. Todos os trabalhos publicados eletronicamente poderão posteriormente ser publicados em coletâneas impressas sob coordenação da Sustenere Publishing, da Companhia Brasileira de Produção Científica e seus parceiros autorizados. Os (as) autores (as) preservam os direitos autorais, mas não têm permissão para a publicação da contribuição em outro meio, impresso ou digital, em português ou em tradução. 